\title{
The Brazilian Legal Amazon Odonatofauna: a perspective of diversity and knowledge gaps
}

\author{
Manoel Daltro Nunes Garcia Junior ${ }^{1 \bowtie}$, Monique Telcia dos Santos Damasceno ${ }^{1 \oplus}$, Diogo Silva Vilela ${ }^{2 \oplus} \&$ \\ Raimundo Nonato Picanço Souto ${ }^{1 \odot}$
}

1. Universidade Federal do Amapá-UNIFAP, Macapá, Amapá, Brazil. 2. Universidade Estadual Paulista, Assis, São Paulo, Brazil.

\section{EntomoBrasilis 15: e977 (2022)}

\begin{abstract}
The Brazilian legal Amazon occupies approximately $61 \%$ of its territory, covering a large part of Brazil's biodiversity. This large territorial dimension generates huge gaps in the animal diversity understanding, for example, the poor knowledge regarding the Odonata order. Worldwide, Odonata has almost 6,500 described species, with approximately 1,800 being recorded for the Neotropical region. Data on the Odonata order in the legal Amazon is still scarce, mainly due to its particularities, and little is known about the diversity of dragonflies in some of Brazilian states. Thus, the objective of this study is to present a list of species occurring in the states that make up the Brazilian legal Amazon. The list was made from the analysis of approximately 165 scientific papers, in addition to occurrence records contained in the $\mathrm{SiBBr}$ and GBIF databases. 641 species were found, which is equivalent to approximately $69 \%$ of the odonatofauna in Brazil. The states with the greatest diversity were Amazonas $(n=364)$, Pará $(n=310)$ and Mato Grosso $(n=285)$. The study also indicated a low level of knowledge of the Odonata order in the states of Tocantins and Maranhão, in addition to the area of the Guianas shields, especially in the states of Amapá and Roraima. Carrying out new inventories and building catalogs is essential for understanding the biodiversity in this region, especially in areas with greater need.
\end{abstract}

Keywords: Anisoptera; Brazil; Inventories; Zygoptera.

Edited by:

Danielle Anjos-Santos

Article History:

Received: 27.x.2021

First Answer: 28.xi.2021

Accepted: $30 . x i i .2021$

Published: 14.ii.2022

Corresponding author:

Manoel Daltro Nunes Garcia Junior

\} m.d.juniorbio@gmail.com

Funding agencies:

$\therefore$ Coordenação de Aperfeiçoamento de Pessoal de Nível Superior (CAPES); Fundação de Amparo à Pesquisa do Amapá (FAPEAP); Fundação de Amparo à Pesquisa do Estado de São Paulo (FAPESP)

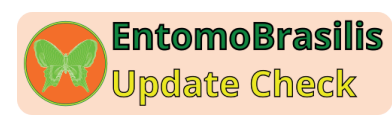

doi: 10.12741/ebrasilis.v15.e977

(c) The Author(s) 2022. Published by Entomologistas do Brasil

This article is published by Entomologistas do Brasil and licensed under Creative Commons Licence 4.0 (CC-BY)

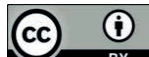

๖ Article Full Open Access
In Brazil, the Legal Amazon encompasses nine states, an area correspondent to approximately $61 \%$ of the country's territory (IBGE 2021). In addition to the Amazon biome, the Cerrado, Pantanal and Caatinga biomes are present in some of the Legal Amazon states that compose this area of Brazil. The Legal Amazon has suffered from the increase of deforestation, where between the years 2018-2019 alone, there was a 34\% increase in the number of deforested areas (INPE 2020). According to MARCOVITCH \& PINSKY (2020), the increase in deforestation rates in the Amazon region is a consequence of the systematic dismantling of environmental policies in Brazil. Also according to MARCOVITCH \& PINSKY (2020), environmental preservation in the Amazon faces another serious problem, which is the poor economic development planning. In addition to deforestation, the major threats to biodiversity in the region are the increase in fires and the fragmentation of forest areas (LAURENCE et al. 2001). This continuous degradation has caused enormous impacts on Brazilian biodiversity, as many species are restricted to the Amazon region (Silva et al. 2005).

Due to its large territorial dimension, the Legal Amazon has huge gaps in the diversity knowledge of many groups, including the Odonata order. Almost 6,500 species of dragonflies are described worldwide (PAULSON et al. 2021), with around 1,800 species only for the Neotropical region (Von Ellenrieder 2009). Considered charismatic insects (CORBET 1999), odonates are easy to observe and handle in the field (DE MARCO \& VIANNA 2005). Even so, only $\sim 29 \%$ of the Brazilian territory has available data regarding this insect order ( $D E$ Marco \& Vianna 2005). According to Juen \& De Marco (2012), many species of Odonata have their distribution limited by a number of factors, which makes understanding the order even more difficult. In addition, due to anthropic actions, such as the removal of vegetation along water bodies, there has been a reduction in some groups, especially representatives of the suborder Zygoptera (De Marco et al. 2015; OliveIRA-Junior \& Juen 2019).

In the Amazon region, despite the growing number of studies published with dragonflies, mainly in the last ten years (CAlvão et al. 2014; NeISS \& HamAda 2014; Juen et al. 2014; Miguel et al. 2017; Alves-Martins et al. 2019; Bastos et al. 2019; Koroiva et al. 2020; Moura et al. 2020; GARCIA JúNIOR et al. 2020, 2021a, 2021 b; BRITO et al. 2021), knowledge concerning the diversity of the order is still scarce. Thus, the objective of this study is to present data from a bibliographic review of Odonata species occurring in the Legal Amazon states. In addition, we aim to indicating areas that, after reviewing the literature, contain little information about the Odonata order.

\section{MATERIAL AND METHODS}

The list of species occurring in the states of the Brazilian legal Amazon was made based on the analysis of approximately 165 scientific papers contained in the open access platforms of the Scientific Electronic Library Online (SciELO) and on the "Coordenação 
de Aperfeiçoamento de Pessoal de Nível Superior" (CAPES Portal). The search for works was carried out using the following descriptors: Amazônia, Odonata, Dragonfly, Damselfly, Amazonian, Zygoptera and Anisoptera, in addition to the names of the nine states that compose the Legal Amazon: Acre, Amapá, Amazonas, Maranhão, Mato Grosso, Pará, Rondônia, Roraima, and Tocantins (Figure 1). We also included in the study: Course completion papers, dissertations, theses and articles written in English and Portuguese that met the used filters used. Finally, we also used data already added to the databases of the Brazilian Biodiversity Information System "Sistema de Informação sobre a Biodiversidade Brasileira" (SIBBR 2021) and the Global Biodiversity Information Facility (GBIF).

\section{RESULTS}

During the present study, 16 doctoral theses, 11 masters dissertations, five course completion papers and 133 scientific articles were analyzed. In addition, were also analyzed the records of 328 species in SiBBR and approximately 230 in the GBIF platform (GBIF 2021). We found records of 641 species, 16 families and 122 genera to the Legal Amazon states (Table 1). Anisoptera, with 340 species inserted in five families, was the suborder with the greatest diversity, followed by Zygoptera with 10 families and 301 species. Among the most representative families in this study are Coenagrionidae, Libellulidae and Gomphidae with 216, 199 and 82 species respectively. These three families totaled approximately $78 \%$ of all species found. Megapodagronidae, Platystictidae and the monotypic family Rimanellidae were the families with the lowest recorded diversity, with only one species each.

In Table 1, it is possible to observe that among the Legal
Amazon states, Amazonas has the largest number of species restricted only to its territory $(n=100)$, followed by Mato Grosso $(n=69)$ and Pará $(n=40)$. Among the genera found with the highest number of species are Erythrodiplax Brauer, $1868(\mathrm{n}=32)$, Micrathyria Kirby, $1889(\mathrm{n}=31)$, Argia Rambur, $1842(n=30)$ and Acanthagrion Selys, $1876(n=25)$. Together, these four genera comprise almost $19 \%$ of the total recorded species.

The greatest diversities were found in the states of Amazonas $(n=364)$, Pará $(n=310)$ and Mato Grosso $(n=285)$ (Table 2). In contrast, Roraima $(n=79)$, and Tocantins $(n=49)$ had the lowest species richness. The number of confirmed occurrences for Amazonas $(n=364)$ represents about $57 \%$ of the species found during the study, while Tocantins, with 49 species, reached only close to $8 \%$ of the total number of records.

\section{DISCUSSION}

By using our search methodology (SciELO, CAPES Portal, $\mathrm{SiBBr}$ and GBIF), 641 species were found with occurrence records for at least one of the states in the Legal Amazon. This number corresponds to approximately 69\% of the odonatofauna recorded for Brazil, which has just over 900 species (PINTO 2020). If we compare only the results of the diversity found in the states of Acre, Amazonas, Amapá, Pará, Rondônia and Roraima, where the Amazon biome fully covers its territories, the number of 545 species is almost 8\% greater than the 503 occurrences cited for the Amazon region by BRASIL et al. (2021).

So far, the odontofauna of Amazonas state had recorded 324 species according to Korolva et al. (2020). From the

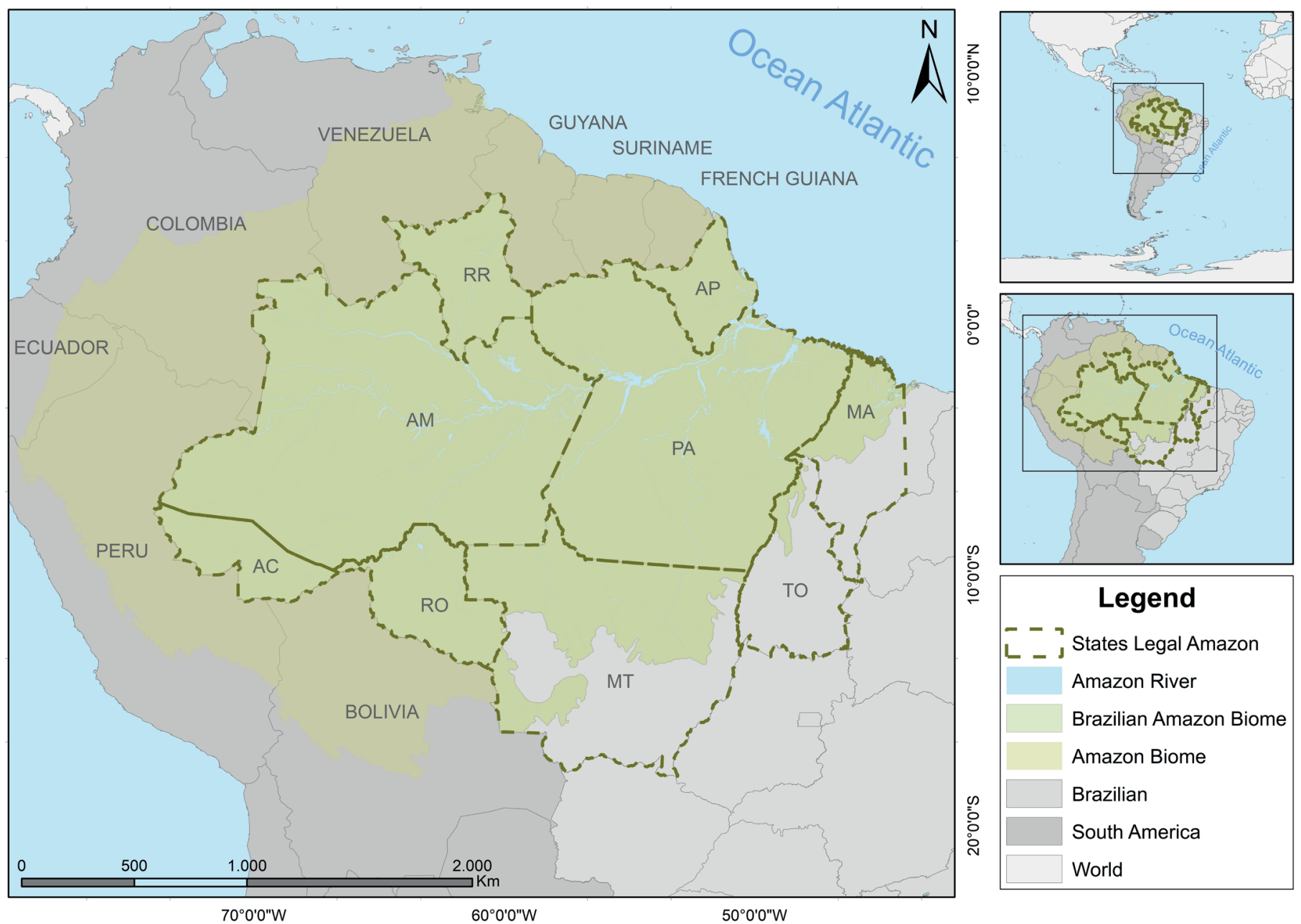

Figure 1. Map of Brazilian Legal Amazon states. Acre- Ac, Amazonas- AM, Amapá- AP, Maranhão- MA, Mato Grosso- MT, ParáPA, Rondônia-RO, Roraima- RR e Tocantins- TO. 
Table 1. List of species occurring in Legal Amazon states. Acre-Ac, Amazonas-AM, Amapá-AP, Maranhão-MA, Mato Grosso-MT, Pará-PA, Rondônia-RO, Roraima-RR and Tocantins-TO.

\begin{tabular}{|c|c|c|}
\hline Suborder/Family & Species & Brazilian Legal Amazon states \\
\hline \multicolumn{3}{|l|}{ Zygoptera } \\
\hline \multicolumn{3}{|l|}{ Calopterygidae } \\
\hline & Hetaerina amazonica Sjöstedt, 1918 & $\mathrm{AM}, \mathrm{AP}, \mathrm{MA}, \mathrm{MT}, \mathrm{PA}, \mathrm{RO}$ \\
\hline & Hetaerina auripennis (Burmeister, 1839) & $\mathrm{AM}, \mathrm{MA}, \mathrm{MT}, \mathrm{PA}, \mathrm{RO}$ \\
\hline & Hetaerina brightwelli (Kirby, 1823) & AM, PA \\
\hline & Hetaerina caja (Drury, 1773) & AM \\
\hline & Hetaerina curvicauda Garrison, 1990 & $\mathrm{MA}, \mathrm{MT}, \mathrm{PA}, \mathrm{RO}$ \\
\hline & Hetaerina fuscoguttata Selys, 1878 & MT \\
\hline & Hetaerina hebe Selys, 1853 & $\mathrm{RO}$ \\
\hline & Hetaerina indeprensa Garrison, 1990 & $A P, P A, R O$ \\
\hline & Hetaerina laesa Hagen in Selys, 1853 & $A C, A M, A P, M T, P A, R O$ \\
\hline & Hetaerina medinai Rácenis, 1968 & AM \\
\hline & Hetaerina moribunda Hagen in Selys, 1853 & $A M, A P, M T, P A, R O$ \\
\hline & Hetaerina mortua Hagen in Selys, 1853 & $A P, R R, R O$ \\
\hline & Hetaerina occisa Hagen in Selys, 1853 & AM \\
\hline & Hetaerina rosea Selys, 1853 & $\mathrm{AM}, \mathrm{MA}, \mathrm{MT}, \mathrm{PA}, \mathrm{RO}$ \\
\hline & Hetaerina sanguinea Selys, 1853 & $A C, A M, M A, P A, R R, R O$ \\
\hline & Hetaerina simplex Selys, 1853 & MA, TO \\
\hline & Hetaerina westfalli Rácenis, 1968 & $\mathrm{AM}, \mathrm{MT}, \mathrm{PA}, \mathrm{RR}, \mathrm{RO}$ \\
\hline & Mnesarete aenea (Selys, 1853) & $A C, A M, M T, P A, R O$ \\
\hline & Mnesarete astrape De Marmels, 1989 & $\mathrm{AM}, \mathrm{PA}$ \\
\hline & Mnesarete cupraea (Selys, 1853) & $A C, A M, P A, M T, R O$ \\
\hline & Mnesarete fuscibasis (Calvert, 1909) & MT \\
\hline & Mnesarete guttifera (Selys, 1873) & MT, TO \\
\hline & Mnesarete lencionii Garrison, 2006 & MT \\
\hline & Mnesarete loutoni Garrison, 2006 & AM \\
\hline & Mnesarete machadoi Garrison, 2006 & MT \\
\hline & Mnesarete pudica (Hagen in Selys, 1853) & MT \\
\hline & Mnesarete smaragdina (Selys, 1869) & $\mathrm{MT}, \mathrm{PA}, \mathrm{RO}$ \\
\hline & Mnesarete williamsoni Garrison, 2006 & MT, PA \\
\hline
\end{tabular}

\section{Coenagrionidae}

Acanthagrion abunae Leonard, 1977

Acanthagrion adustum Williamson, 1916

Acanthagrion aepiolum Tennessen, 2004

Acanthagrion amazonicum Sjöstedt, 1918

Acanthagrion ascendens Calvert, 1909

Acanthagrion apicale Selys, 1876

Acanthagrion chacoense Calvert, 1909

Acanthagrion chararum Calvert, 1909

Acanthagrion cuyabae Calvert, 1909

Acanthagrion egleri (Santos, 1961)

Acanthagrion flaviae Machado, 2012

Acanthagrion gracile (Rambur, 1842)

Acanthagrion hildegarda Gloger, 1967

Acanthagrion jessei Leonard, 1977

Acanthagrion Kaori Machado, 2012

Acanthagrion kennedii Williamson, 1916

Acanthagrion longispinosum Leonard, 1977

Acanthagrion minutum Leonard, 1977

Acanthagrion floridense Fraser, 1946

Acanthagrion phallicorne Leonard, 1977

\section{$A P, M T, R O$}

$A M, A P, P A, R R, R O$

$M A, P A, R O$

$A M, A P, M A, P A, R O$

$A P, M T, P A, R O$

$A C, A M, A P, M T, P A, R R, R O$

MT

AM, MT

AM, MT, RO

$A P, P A$

AM

$A C, M A, M T, P A, R R$

TO

MT, PA, RO

AM

$A P, M A, P A$

$A M, R O$

AM, MT, PA

RO

AM, AP, MT, PA, RO

To be continue... 
Table 1. Continue...

\begin{tabular}{|c|c|c|}
\hline Suborder/Family & Species & Brazilian Legal Amazon states \\
\hline \multirow[t]{50}{*}{ Coenagrionidae } & Acanthagrion quadratum Selys, 1876 & AM \\
\hline & Acanthagrion rubrifrons Leonard, 1977 & $\mathrm{AM}, \mathrm{PA}$ \\
\hline & Acanthagrion temporale Selys, 1876 & $\mathrm{MT}, \mathrm{RO}$ \\
\hline & Acanthagrion tepuiense De Marmels, 1985 & AM \\
\hline & Acanthagrion truncatum Selys, 1876 & $A P, M A, M T, P A, T O$ \\
\hline & Acanthallagma caeruleum Williamson \& Williamson, 1924 & AM \\
\hline & Acanthallagma luteum Williamson \& Williamson, 1924 & $\mathrm{PA}, \mathrm{RO}$ \\
\hline & Acanthallagma strohmi Williamson \& Williamson, 1924 & RO \\
\hline & Aceratobasis macilenta (Rambur, 1842) & $A P, P A$ \\
\hline & Aeolagrion dorsale (Burmeister, 1839) & $A P, A M, P A, R O$ \\
\hline & Aeolagrion inca (Selys, 1876) & AM \\
\hline & Amazoneura ephippigera (Selys, 1886) & AM \\
\hline & Amazoneura juruaensis Machado, 2004 & $A C$ \\
\hline & $\begin{array}{l}\text { Argia angelae Vilela, Guillermo-Ferreira, Del-Claro \& Cordero- } \\
\text { Rivera, } 2018\end{array}$ & MT \\
\hline & Argia bicellulata (Calvert, 1909) & $\mathrm{AM}, \mathrm{MT}$ \\
\hline & Argia botacudo Calvert, 1909 & AM, MT \\
\hline & Argia chapadae Calvert, 1909 & MT, PA, TO \\
\hline & Argia collata Selys, 1865 & $\mathrm{AM}, \mathrm{PA}$ \\
\hline & Argia croceipennis Selys, 1865 & MT, PA \\
\hline & Argia cuneifera Garrison \& von Ellenrieder, 2015 & $\mathrm{AM}$ \\
\hline & Argia euphorbia Fraser, 1946 & $A C, A M, P A, R O$ \\
\hline & Argia fumigata Hagen in Selys, 1865 & $A M, A P, P A, R O$ \\
\hline & Argia gemella Garrison \& Von Ellenrieder, 2015 & $\mathrm{AM}, \mathrm{AP}$ \\
\hline & Argia hasemani Calvert, 1909 & AM, MA, MT, PA \\
\hline & Argia impura Rambur, 1842 & AM \\
\hline & Argia indicatrix Calvert, 1901 & $\mathrm{AM}, \mathrm{AP}, \mathrm{MA}, \mathrm{PA}, \mathrm{RO}$ \\
\hline & Argia infumata Selys, 1865 & $A C, A M, P A, R O$ \\
\hline & Argia insipida Hagen in Selys, 1865 & $\mathrm{AM}, \mathrm{MT}, \mathrm{PA}$ \\
\hline & Argia lilacina Selys, 1865 & MA, MT, PA, TO \\
\hline & Argia loutoni Garrison \& von Ellenrieder, 2015 & AM \\
\hline & Argia meioura Garrison \& von Ellenrieder, 2015 & AM \\
\hline & Argia modesta Selys, 1865 & PA \\
\hline & Argia mollis Hagen in Selys, 1865 & $\mathrm{AM}, \mathrm{MT}, \mathrm{PA}, \mathrm{RO}$ \\
\hline & Argia oculata Hagen in Selys, 1865 & $A M, A P, M T, P A, R O$ \\
\hline & Argia palmata Garrison \& von Ellenrieder, 2015 & $\mathrm{AM}, \mathrm{MA}$ \\
\hline & Argia pulla Hagen in Selys, 1865 & $A M, A P, R R$ \\
\hline & Argia reclusa Selys, 1865 & $A M, M A, M T, P A$ \\
\hline & Argia smithiana Calvert, 1909 & $\mathrm{MT}, \mathrm{PA}, \mathrm{RO}$ \\
\hline & Argia subapicalis Calvert, 1909 & $\mathrm{AM}, \mathrm{MT}$ \\
\hline & Argia tamoyo Calvert, 1909 & MT \\
\hline & Argia thespis Hagen in Selys, 1865 & $\mathrm{AM}, \mathrm{PA}, \mathrm{RO}, \mathrm{TO}$ \\
\hline & Argia tinctipennis Selys, 1865 & $\mathrm{AM}, \mathrm{MT}, \mathrm{PA}, \mathrm{RO}$ \\
\hline & Argia translata Hagen in Selys, 1865 & $A M, A P$ \\
\hline & Argia tupi Calvert, 1909 & MT, PA \\
\hline & Bromeliagrion beebeanum (Calvert, 1948) & AM \\
\hline & Bromeliagrion rehni Garrison, 2005 & AM \\
\hline & Calvertagrion charis Tennessen, 2015 & AM \\
\hline & Calvertagrion minutissimum (Selys, 1876) & $A M, P A, R O$ \\
\hline & Carajathemis simone Machado, 2012 & PA \\
\hline & Cyanallagma ferenigrum De Marmels, 2003 & MT, PA, TO \\
\hline
\end{tabular}


Table 1. Continue...

\begin{tabular}{|c|c|c|}
\hline Suborder/Family & Species & Brazilian Legal Amazon states \\
\hline \multirow[t]{51}{*}{ Coenagrionidae } & Denticulobasis ariken Machado, 2009 & $\mathrm{AM}, \mathrm{RO}$ \\
\hline & Denticulobasis garrisoni Machado, 2009 & $\mathrm{RO}$ \\
\hline & Drepanoneura janirae Von Ellenrieder \& Garrison, 2008 & RO \\
\hline & Drepanoneura muzoni Von Ellenrieder \& Garrison, 2008 & AM \\
\hline & Dolonagrion fulvellum (Selys, 1876) & $A M, A P$ \\
\hline & Epipleoneura albuquerquei Machado, 1964 & $A M, P A, R O$ \\
\hline & Epipleoneura angeloi Pessacq \& Costa, 2010 & MT, TO \\
\hline & Epipleoneura capilliformis (Selys, 1886) & $\mathrm{AM}, \mathrm{AP}, \mathrm{MT}, \mathrm{PA}$ \\
\hline & Epipleoneura demarmelsi Von Ellenrieder \& Garrison, 2008 & AM \\
\hline & Epipleoneura fuscaenea Williams, 1915 & $\mathrm{AM}, \mathrm{PA}$ \\
\hline & Epipleoneura haroldoi Santos, 1964 & $\mathrm{AM}, \mathrm{PA}$ \\
\hline & Epipleoneura humeralis (Selys, 1886) & AM, PA \\
\hline & Epipleoneura janirae Machado, 2005 & AM, PA \\
\hline & Epipleoneura kaxuriana Machado, 1985 & $\mathrm{AM}, \mathrm{AP}, \mathrm{PA}, \mathrm{RO}$ \\
\hline & Epipleoneura lamina Williamson, 1915 & $A M, P A, R O$ \\
\hline & Epipleoneura machadoi Rácenis, 1960 & MT, PA, TO \\
\hline & Epipleoneura manauensis Santos, 1964 & $\mathrm{AM}, \mathrm{PA}$ \\
\hline & Epipleoneura metallica Rácenis, 1955 & AM, MA, MT, PA, RO, TO \\
\hline & Epipleoneura ocuene De Marmels, 1989 & PA \\
\hline & Epipleoneura pereirai Machado, 1964 & $\mathrm{AP}, \mathrm{PA}$ \\
\hline & Epipleoneura solitaria De Marmels, 1989 & PA \\
\hline & Epipleoneura spatulata Rácenis, 1960 & $\mathrm{AM}, \mathrm{PA}$ \\
\hline & Epipleoneura susanae Pessacq, 2014 & MT \\
\hline & Epipleoneura tariana Machado, 1985 & $A C, A M, P A$ \\
\hline & Epipleoneura uncinata De Marmels, 1989 & AM \\
\hline & Epipleoneura venezuelensis Rácenis, 1955 & $A P, M T, M A, P A, R O$ \\
\hline & Epipleoneura waiwaiana Machado, 1985 & $\mathrm{AM}, \mathrm{PA}$ \\
\hline & Epipleoneura westfalli Machado, 1986 & $\mathrm{MA}, \mathrm{MT}, \mathrm{PA}, \mathrm{RO}$ \\
\hline & Epipleoneura williamsoni Santos, 1957 & MA, MT, PA, TO \\
\hline & Epipotoneura machadoi Von Ellenrieder \& Garrison, 2008 & PA \\
\hline & Epipotoneura nehalennia Williamson, 1915 & AM \\
\hline & Forcepsioneura itatiaiae Santos, 1970 & AM \\
\hline & Helveciagrion obsoletum (Selys, 1876) & MT \\
\hline & Homeoura nepos (Selys, 1876) & $\mathrm{AM}, \mathrm{AP}, \mathrm{MT}, \mathrm{PA}, \mathrm{RO}$ \\
\hline & Homeoura obrieni Von Ellenrieder, 2008 & AM \\
\hline & Hylaeonympha magoi Rácenis, 1968 & AM \\
\hline & Inpabasis machadoi Santos, 1961 & $A M, P A, R O$ \\
\hline & Inpabasis rosea (Selys, 1877) & $\mathrm{AM}, \mathrm{AP}$ \\
\hline & Ischnura capreolus (Hagen, 1861) & $A C, A M, A P, M A, M T, P A, R R$ \\
\hline & Ischnura fluviatilis Selys, 1876 & $\mathrm{AM}, \mathrm{AP}, \mathrm{MT}, \mathrm{PA}, \mathrm{RR}$ \\
\hline & Ischnura hastata (Say, 1840) & $\mathrm{RR}$ \\
\hline & Leptagrion aculeatum Santos, 1965 & $\mathrm{AP}, \mathrm{PA}$ \\
\hline & Leucobasis candicans Rácenis, 1959 & AM \\
\hline & Mecistogaster amalia (Burmeister, 1839) & $A C, A M, A P, M T, P A, R O, R R$ \\
\hline & Mecistogaster lucretia (Drury, 1773) & $\mathrm{AM}, \mathrm{AP}, \mathrm{MT}, \mathrm{PA}$ \\
\hline & Mecistogaster ornata Rambur, 1842 & $A M, M T, P A, R O, R R$ \\
\hline & Mesoleptobasis acuminata Santos, 1961 & $\mathrm{AM}, \mathrm{PA}, \mathrm{MT}, \mathrm{RO}$ \\
\hline & Mesoleptobasis cantralli Santos, 1961 & $\mathrm{AM}, \mathrm{RO}$ \\
\hline & Mesoleptobasis elongata Garrison \& von Ellenrieder, 2009 & $\mathrm{AM}, \mathrm{RR}$ \\
\hline & Mesoleptobasis incus Sjöstedt, 1918 & $\mathrm{AM}, \mathrm{RO}$ \\
\hline & Metaleptobasis amazonica Sjöstedt, 1918 & AM \\
\hline
\end{tabular}


Table 1. Continue...

\begin{tabular}{|c|c|c|}
\hline Suborder/Family & Species & Brazilian Legal Amazon states \\
\hline \multirow[t]{51}{*}{ Coenagrionidae } & Metaleptobasis bicornis (Selys, 1877) & $A M, P A, R R$ \\
\hline & Metaleptobasis brysonima Williamson, 1915 & AM \\
\hline & Metaleptobasis diceras (Selys, 1877) & AP, MT, PA, RO, RR, TO \\
\hline & Metaleptobasis falcifera Von Ellenrieder, 2013 & $A C$ \\
\hline & Metaleptobasis inermis Von Ellenrieder, 2013 & $A C$ \\
\hline & Metaleptobasis leniloba Von Ellenrieder, 2013 & PA \\
\hline & Metaleptobasis lillianae Daigle, 2004 & MT \\
\hline & Metaleptobasis longicauda Von Ellenrieder, 2013 & MT \\
\hline & Metaleptobasis minteri Daigle, 2003 & $A C$ \\
\hline & Metaleptobasis paludicola Von Ellenrieder, 2013 & AM \\
\hline & Metaleptobasis quadricornis (Selys, 1877) & RO \\
\hline & Metaleptobasis selysi Santos, 1956 & MT, RO \\
\hline & Metaleptobasis spatulata Von Ellenrieder, 2013 & RO \\
\hline & Metaleptobasis tridentigera Von Ellenrieder, 2013 & $A M, P A, R O$ \\
\hline & Metaleptobasis truncata Von Ellenrieder, 2013 & PA \\
\hline & Microstigma anomalum Rambur, 1842 & $A C, A M, P A, R O$ \\
\hline & Microstigma maculatum Hagen in Selys, 1860 & $A M, A P, P A, R O$ \\
\hline & Microstigma rotundatum Selys, 1860 & $A C, A M, A P, P A$ \\
\hline & Minagrion canaanense (Santos, 1967) & PA \\
\hline & Minagrion waltheri (Selys, 1876) & TO \\
\hline & Nehalennia minuta (Selys in Sagra, 1857) & AP \\
\hline & Neoneura bilinearis Selys, 1860 & $A C, A M, A P, M T, P A, R R, R O$ \\
\hline & Neoneura denticulata Williamson, 1917 & $A C, A M, M T, P A, R R, R O$ \\
\hline & Neoneura desana Machado, 1989 & $\mathrm{AM}, \mathrm{AP}$ \\
\hline & Neoneura ethela Williamson, 1917 & MA \\
\hline & Neoneura fulvicollis Selys, 1886 & MA, MT, PA \\
\hline & Neoneura joana Williamson, 1917 & $A P, P A, R O, R R$ \\
\hline & Neoneura lucas Machado, 2002 & MT, PA \\
\hline & Neoneura luzmarina De Marmels, 1989 & AM, MT, PA \\
\hline & Neoneura mariana Williamson, 1917 & AM \\
\hline & Neoneura moorei Machado, 2003 & RO \\
\hline & Neoneura myrthea Williamson, 1917 & $A M, A P, P A, R R$ \\
\hline & Neoneura rubriventris Selys, 1860 & $A M, A P, P A, R R, R O$ \\
\hline & Neoneura rufithorax Selys, 1886 & $A C, A M, P A, R O$ \\
\hline & Neoneura schreiberi Machado, 1975 & AP \\
\hline & Neoneura sylvatica Hagen in Selys, 1886 & $A P, M A, M T, P A, R R, R O, T O$ \\
\hline & Oxyagrion basale Selys, 1876 & MT \\
\hline & Oxyagrion chapadense Costa, 1978 & MT, TO \\
\hline & Oxyagrion evanescens Calvert, 1909 & MT, TO \\
\hline & Oxyagrion fernandoi Costa, 1988 & MT, PA \\
\hline & Oxyagrion impunctatum Calvert, 1909 & MT, TO \\
\hline & Oxyagrion microstigma Selys, 1876 & MT \\
\hline & Oxyagrion sulmatogrossense Costa, Souza e Santos, 2000 & MT \\
\hline & Oxyagrion pavidum Hagen, 1876 & MT \\
\hline & Oxyagrion terminale Selys, 1876 & AM, PA \\
\hline & Peristicta aeneoviridis Calvert, 1909 & MT \\
\hline & Peristicta forceps Hagen in Selys, 1860 & MT \\
\hline & Peristicta muzoni Pessacq \& Costa, 2007 & MT \\
\hline & Phasmoneura exigua (Selys, 1886) & $A M, A P, M T, P A$ \\
\hline & Phasmoneura janirae Lencioni, 1999 & MT, PA \\
\hline & Phoenicagrion flammeum (Selys, 1876) & $A C, A M, A P, M T, P A, R O, R R$ \\
\hline
\end{tabular}


Table 1. Continue...

\begin{tabular}{|c|c|c|}
\hline Suborder/Family & Species & Brazilian Legal Amazon states \\
\hline \multirow{45}{*}{ Coenagrionidae } & Phoenicagrion flavescens Machado, 2010 & AP, PA \\
\hline & Phoenicagrion ibseni Machado, 2010 & AP \\
\hline & Phoenicagrion karaja Machado, 2010 & MT, PA \\
\hline & Phoenicagrion megalobos Machado, 2010 & PA \\
\hline & Platystigma buckleyi (McLachlan, 1881) & AM \\
\hline & Platystigma jocaste (Hagen, 1869) & $A C, R O$ \\
\hline & Protoneura amatoria Calvert, 1907 & AM, RR \\
\hline & Protoneura paucinervis Selys, 1886 & MT \\
\hline & Protoneura scintilla Gloyd, 1939 & $A M, A P, P A, R O$ \\
\hline & Protoneura tenuis Selys, 1860 & $A C, A M, M T, P A, R R, R O$ \\
\hline & Psaironeura bifurcata (Sjöstedt, 1918) & $\mathrm{AC}, \mathrm{AM}$ \\
\hline & Psaironeura tenuissima (Selys, 1886) & $A M, A P, P A, R O$ \\
\hline & Telebasis abuna Bick \& Bick, 1995 & $\mathrm{AM}, \mathrm{MA}, \mathrm{RO}$ \\
\hline & Telebasis carmesina Calvert, 1909 & AC, MT \\
\hline & Telebasis carminita Calvert, 1909 & $A P, M T, P A, R R$ \\
\hline & Telebasis carvalhoi Garrison, 2009 & PA \\
\hline & Telebasis celiovallei Machado, 2010 & PA \\
\hline & Telebasis coccinea (Selys, 1876) & MA, MT, PA, TO \\
\hline & Telebasis corallina (Selys, 1876) & $P A, R R$ \\
\hline & Telebasis corbeti Garrison, 2009 & AC \\
\hline & Telebasis demarara (Williamson, 1917) & AM, MA, PA \\
\hline & Telebasis divaricata Machado, 2010 & PA, TO \\
\hline & Telebasis dunklei Bick \& Bick, 1995 & AM \\
\hline & Telebasis filiola (Perty, 1833) & MA, MT, PA \\
\hline & Telebasis griffinii (Martin, 1896) & $A C, A M, A P, M T, P A, R O$ \\
\hline & Telebasis inalata (Calvert, 1961) & AM \\
\hline & Telebasis lenkoi Machado, 2010 & MT \\
\hline & Telebasis leptocyclia Garrison, 2009 & $\mathrm{RO}$ \\
\hline & Telebasis obsoleta (Selys, 1876) & $A C, A M, P A, M T$ \\
\hline & Telebasis racenisi Bick \& Bick, 1995 & MT, PA, RO, TO \\
\hline & Telebasis rubricauda Bick \& Bick, 1995 & $\mathrm{AC}, \mathrm{RO}$ \\
\hline & Telebasis sanguinalis Calvert, 1909 & AM, MT, PA \\
\hline & Telebasis simulacrum (Calvert, 1909) & MT \\
\hline & Telebasis simulata Tennessen, 2002 & AM, PA \\
\hline & Telebasis vulcanoae (Machado, 1980) & PA \\
\hline & Telebasis willinki Fraser, 1984 & MT \\
\hline & Tigriagrion aurantinigrum Calvert, 1909 & $A C, M A, M T, P A, R O$ \\
\hline & Tuberculobasis arara Machado, 2009 & RO \\
\hline & Tuberculobasis inversa (Selys, 1876) & $A M, P A, R O$ \\
\hline & Tuberculobasis karitiana Machado, 2009 & RO \\
\hline & Tuberculobasis macuxi Machado, 2009 & $\mathrm{RR}$ \\
\hline & Tuberculobasis mammilaris (Calvert, 1909) & MT \\
\hline & Tuberculobasis tirio Machado, 2009 & PA \\
\hline & Tuberculobasis yanomami (De Marmels, 1992) & PA \\
\hline & Tukanobasis corbeti Machado, 2009 & AM \\
\hline \multicolumn{3}{|l|}{ Dicteriadidae } \\
\hline & Dicterias atrosanguinea Selys, 1853 & AM, PA \\
\hline
\end{tabular}

\section{Dicteriadidae}

\section{AM, PA}

Heliocharis amazona Selys, 1853

$A C, A M, A P, M T, P A, R O$ 
Table 1. Continue...

\begin{tabular}{|c|c|c|}
\hline Suborder/Family & Species & Brazilian Legal Amazon states \\
\hline \multirow[t]{13}{*}{ Heteragrionidae } & Heteragrion angustipenne Selys, 1886 & AM, RO \\
\hline & Heteragrion aurantiacum Selys, 1862 & PA \\
\hline & Heteragrion bariai De Marmels, 1989 & $A C, A M, P A, R O$ \\
\hline & Heteragrion cinnamomeum Selys, 1862 & AP \\
\hline & Heteragrion consors Hagen in Selys, 1862 & AM \\
\hline & Heteragrion ictericum Williamson, 1919 & $A M, A P, P A, R O$ \\
\hline & Heteragrion icterops Selys, 1862 & $\mathrm{AM}, \mathrm{MT}, \mathrm{PA}, \mathrm{RO}$ \\
\hline & Heteragrion inca Calvert, 1909 & $\mathrm{AM}, \mathrm{RO}$ \\
\hline & Heteragrion silvarum Sjöstedt, 1918 & $A M, P A, R O$ \\
\hline & Heteragrion simulatum Williamson, 1919 & AM \\
\hline & Heteragrion triangulare Calvert, 1901 & MT \\
\hline & Oxystigma cyanofrons Williamson, 1919 & $\mathrm{AM}, \mathrm{AP}$ \\
\hline & Oxystigma petiolatum (Selys, 1862) & $\mathrm{AM}, \mathrm{AP}, \mathrm{PA}, \mathrm{RR}, \mathrm{RO}$ \\
\hline \multicolumn{3}{|l|}{ Lestidae } \\
\hline & Lestes bipupillatus Calvert, 1909 & MT \\
\hline & Lestes dichrostigma Calvert, 1909 & MT \\
\hline & Lestes falcifer Sjöstedt, 1918 & $A M, R R$ \\
\hline & Lestes fernandoi Costa, De Souza \& Muzón, 2006 & MA \\
\hline & Lestes forficula Rambur, 1842 & $\mathrm{MA}, \mathrm{MT}, \mathrm{RO}, \mathrm{RR}$ \\
\hline & Lestes helix Ris, 1918 & RO \\
\hline & Lestes jurzitzai Muzón, 1994 & RO \\
\hline & Lestes minutus Selys, 1862 & $\mathrm{MT}, \mathrm{RR}$ \\
\hline & Lestes paulistus Calvert, 1909 & MT \\
\hline & Lestes pictus Hagen in Selys, 1862 & MT \\
\hline & Lestes quadristriatus Calvert, 1909 & MT \\
\hline & Lestes tricolor Erichson, 1848 & AM \\
\hline \multicolumn{3}{|l|}{ Megapodagrionidae } \\
\hline & Megapodagrion megalopus (Selys, 1862) & $\mathrm{AM}, \mathrm{MT}, \mathrm{PA}, \mathrm{RO}$ \\
\hline \multicolumn{3}{|l|}{ Perilestidae } \\
\hline & Perilestes attenuatus Selys, 1886 & $A M, P A, R O$ \\
\hline & Perilestes bispinus Kimmins, 1958 & AM \\
\hline & Perilestes fragilis Hagen in Selys, 1862 & AM, PA \\
\hline & Perilestes gracillimus Kennedy, 1941 & $A M, A P, P A$ \\
\hline & Perilestes kahli Williamson \& Williamson, 1924 & $\mathrm{PA}, \mathrm{RO}$ \\
\hline & Perilestes minor Williamson \& Williamson, 1924 & RO \\
\hline & Perilestes solutus Williamson \& Williamson, 1924 & $\mathrm{AM}, \mathrm{MA}, \mathrm{MT}, \mathrm{PA}, \mathrm{RR}, \mathrm{RO}$ \\
\hline & Perissolestes aculeatus Kennedy, 1941 & $\mathrm{RO}$ \\
\hline & Perissolestes cornutus (Selys, 1886) & $\mathrm{AM}, \mathrm{AP}, \mathrm{PA}, \mathrm{RO}$ \\
\hline & Perissolestes flinti De Marmels, 1989 & AM \\
\hline & Perissolestes paprzyckii Kennedy, 1941 & $\mathrm{AC}, \mathrm{AM}$ \\
\hline \multicolumn{3}{|l|}{ Philogeniidae } \\
\hline & Philogenia margarita Selys, 1862 & AM \\
\hline & Philogenia silvarum Ris, 1918 & AM \\
\hline \multicolumn{3}{|l|}{ Platystictidae } \\
\hline & Palaemnema brasiliensis Machado, 2009 & AM, AP \\
\hline \multicolumn{3}{|l|}{ Polythoridae } \\
\hline & Chalcopteryx machadoi Costa, 2005 & PA \\
\hline & Chalcopteryx radians Ris, 1914 & AM, PA \\
\hline & Chalcopteryx rutilans (Rambur, 1842) & $A C, A M, M T, P A, R O$ \\
\hline & Chalcopteryx scintillans McLachlan, 1870 & $\mathrm{AM}, \mathrm{RO}$ \\
\hline & Chalcopteryx seabrai Santos \& Machado, 1861 & $A M, A P$ \\
\hline
\end{tabular}


Table 1. Continue...

\begin{tabular}{|c|c|c|}
\hline Suborder/Family & Species & Brazilian Legal Amazon states \\
\hline \multirow[t]{8}{*}{ Polythoridae } & Euthore fasciata (Hagen in Selys, 1853) & AM \\
\hline & Polythore aurora (Selys, 1879) & AM \\
\hline & Polythore batesi (Selys, 1869) & AM \\
\hline & Polythore beata (McLachlan, 1869) & AM \\
\hline & Polythore manua Bick and Bick, 1990 & $\mathrm{AC}$ \\
\hline & Polythore picta (Rambur, 1842) & $A C, A M, P A$ \\
\hline & Polythore procera (Selys, 1869) & AM \\
\hline & Polythore vittata (Selys, 1869) & $A C, A M, R O$ \\
\hline \multicolumn{3}{|l|}{ Rimanellidae } \\
\hline & Rimanella arcana (Needham, 1933) & AM \\
\hline \multicolumn{3}{|l|}{ Anisoptera } \\
\hline \multicolumn{3}{|l|}{ Aeshnidae } \\
\hline & Aeshna williamsoniana Calvert, 1905 & MT \\
\hline & Anax amazili (Burmeister, 1839) & AM \\
\hline & Anax concolor Brauer, 1865 & AM, MT \\
\hline & Anax longipes Hagen, 1861 & MT \\
\hline & Castoraeschna corbeti Carvalho, Pinto \& Ferreira, 2009 & PA \\
\hline & Castoraeschna januaria (Hagen, 1867) & MT \\
\hline & Castoraeschna longfieldae (Kimmins, 1929) & MT \\
\hline & Castoraeschna tepuica De Marmels, 1989 & AM \\
\hline & Coryphaeschna adnexa (Hagen, 1861) & $A C, A M, A P, M T, P A$ \\
\hline & Coryphaeschna amazonica De Marmels, 1989 & $A M, P A, R R$ \\
\hline & Coryphaeschna viriditas Calvert, 1952 & $\mathrm{AM}, \mathrm{MT}, \mathrm{RR}$ \\
\hline & Gynacantha auricularis Martin, 1909 & $\mathrm{AM}, \mathrm{MT}, \mathrm{PA}, \mathrm{RO}$ \\
\hline & Gynacantha bifida Rambur, 1842 & $A M, P A, R O$ \\
\hline & Gynacantha chelifera McLachlan, 1895 & MT \\
\hline & Gynacantha dryadula Neiss \& De Marmels, 2017 & AM \\
\hline & Gynacantha gracilis (Burmeister, 1839) & $A M, P A, R O$ \\
\hline & Gynacantha interioris Williamson, 1923 & $\mathrm{AC}, \mathrm{AM}, \mathrm{MT}$ \\
\hline & Gynacantha klagesi Williamson, 1923 & AM, PA \\
\hline & Gynacantha litoralis Williamson, 1923 & $A M, P A, R O$ \\
\hline & Gynacantha membranalis Karsch, 1891 & $\mathrm{AM}, \mathrm{AP}, \mathrm{MT}, \mathrm{PA}, \mathrm{RO}$ \\
\hline & Gynacantha mexicana Selys, 1868 & $A M, A P, P A, R R$ \\
\hline & Gynacantha nervosa Rambur, 1842 & $\mathrm{AM}, \mathrm{AP}, \mathrm{MT}, \mathrm{RR}, \mathrm{RO}$ \\
\hline & Gynacantha tenuis Martin, 1909 & AM \\
\hline & Neuraeschna calverti Kimmins, 1951 & $A C, A M, M A$ \\
\hline & Neuraeschna capillata Machet, 1990 & AM \\
\hline & Neuraeschna claviforcipata Martin, 1909 & $\mathrm{AM}, \mathrm{PA}$ \\
\hline & Neuraeschna costalis (Burmeister, 1839) & $A M, A P, P A$ \\
\hline & Neuraeschna dentigera Martin, 1909 & AM, PA \\
\hline & Neuraeschna harpya Martin, 1909 & AM, PA \\
\hline & Neuraeschna maxima Belle, 1989 & PA \\
\hline & Neuraeschna mina Williamson \& Williamson, 1930 & $\mathrm{RO}$ \\
\hline & Neuraeschna producta Kimmins, 1933 & PA \\
\hline & Neuraeschna tapajonica Machado, 2002 & PA \\
\hline & Rhionaeschna planaltica (Calvert, 1952) & $\mathrm{AM}, \mathrm{MT}$ \\
\hline & Remartinia luteipennis (Burmeister, 1839) & $\mathrm{AC}, \mathrm{MT}, \mathrm{PA}$ \\
\hline & Staurophlebia gigantula Martin, 1909 & AM \\
\hline & Staurophlebia reticulata (Burmeister, 1839) & $A C, A P, M T, P A$ \\
\hline & Staurophlebia wayana Geijskes, 1959 & $\mathrm{AM}, \mathrm{MT}$ \\
\hline
\end{tabular}

To be continue... 
Table 1. Continue...

\begin{tabular}{|c|c|c|}
\hline Suborder/Family & Species & Brazilian Legal Amazon states \\
\hline \multirow[t]{6}{*}{ Aeshnidae } & Triacanthagyna caribbea Williamson, 1923 & AM \\
\hline & Triacanthagyna dentata (Geijskes, 1943) & $\mathrm{AM}, \mathrm{RO}$ \\
\hline & Triacanthagyna ditzleri Williamson, 1923 & AM, AP \\
\hline & Triacanthagyna satyrus (Martin, 1909) & AM \\
\hline & Triacanthagyna septima (Selys in Sagra, 1857) & $A M, A P, M T, P A, R R$ \\
\hline & Triacanthagyna trifida (Rambur, 1842) & AM, MT \\
\hline \multicolumn{3}{|l|}{ Corduliidae } \\
\hline & Aeschnosoma auripennis Geijskes, 1970 & AM, MT \\
\hline & Aeschnosoma elegans Selys, 1871 & PA \\
\hline & Aeschnosoma forcipula Hagen in Selys, 1871 & AM, MT, PA \\
\hline & Aeschnosoma hamadae Fleck \& Neiss, 2012 & AM \\
\hline & Aeschnosoma louissiriusi Fleck, 2012 & RO \\
\hline & Navicordulia amazonica Machado \& Costa, 1995 & MT \\
\hline & Navicordulia errans (Calvert, 1909) & MT, TO \\
\hline & Paracordulia sericea (Selys, 1871) & AM \\
\hline \multicolumn{3}{|l|}{ Gomphidae } \\
\hline & Agriogomphus sylvicola Selys, 1869 & AM \\
\hline & Aphylla barbata Belle, 1994 & AM \\
\hline & Aphylla brasiliensis Belle, 1970 & AM, PA, TO \\
\hline & Aphylla brevipes Selys, 1854 & $\mathrm{PA}$ \\
\hline & Aphylla caudalis Belle, 1987 & PA \\
\hline & Aphylla dentata Selys, 1859 & AM, MT, PA \\
\hline & Aphylla distinguenda Campion, 1920 & MT \\
\hline & Aphylla edentata Selys, 1869 & AM \\
\hline & Aphylla exilis Belle, 1994 & PA \\
\hline & Aphylla janirae 1994 & AP \\
\hline & Aphylla linea Belle, 1994 & MT \\
\hline & Aphylla molossus Selys, 1859 & $\mathrm{AM}, \mathrm{PA}$ \\
\hline & Aphylla producta Selys, 1854 & AP \\
\hline & Aphylla theodorina (Navás, 1933) & MA \\
\hline & Aphylla scapula Belle, 1992 & $\mathrm{RO}$ \\
\hline & Archaeogomphus nanus Needham, 1944 & $\mathrm{RR}, \mathrm{TO}$ \\
\hline & Archaeogomphus vanbrinkae Machado, 1994 & MT \\
\hline & Cacoides latro (Erichson in Schomburgk, 1848) & $\mathrm{AM}, \mathrm{PA}$ \\
\hline & Cyanogomphus comparabilis Belle, 1994 & MT \\
\hline & Cyanogomphus waltheri Selys, 1873 & MT \\
\hline & Desmogomphus tigrivensis Williamson, 1920 & AM \\
\hline & Diaphlebia angustipennis Selys, 1854 & $\mathrm{AM}, \mathrm{MT}, \mathrm{PA}, \mathrm{RO}$ \\
\hline & Diaphlebia nexans Calvert, 1903 & MT, TO \\
\hline & Ebegomphus schroederi (Belle, 1970) & PA \\
\hline & Ebegomphus demerarae (Selys, 1894) & MA \\
\hline & Epigomphus hylaeus Ris, 1918 & AM, MT, PA \\
\hline & Gomphidia kirschii Selys, 1878 & PA \\
\hline & Gomphoides infumata (Rambur, 1842) & AM \\
\hline & Idiogomphoides emmeli Belle, 1995 & RO \\
\hline & Melanocacus mungo (Needham, 1940) & $\mathrm{AM}, \mathrm{MT}, \mathrm{RR}$ \\
\hline & Peruviogomphus bellei Machado, 2005 & AM \\
\hline & Phyllocycla armata Belle, 1977 & AM, MT, PA \\
\hline & Phyllocycla bartica Calvert, 1948 & PA \\
\hline & Phyllocycla brasilia Belle, 1988 & MA \\
\hline & Phyllocycla diphylla (Selys, 1854) & AM \\
\hline
\end{tabular}

To be continue... 
Table 1. Continue...

\begin{tabular}{|c|c|c|}
\hline Suborder/Family & Species & Brazilian Legal Amazon states \\
\hline \multirow[t]{48}{*}{ Gomphidae } & Phyllocycla hamata Belle, 1990 & MT, RO \\
\hline & Phyllocycla medusa Belle, 1988 & PA \\
\hline & Phyllocycla modesta Belle, 1970 & $\mathrm{AM}, \mathrm{AP}$ \\
\hline & Phyllocycla neotropica Belle, 1970 & AM \\
\hline & Phyllocycla ophis (Selys, 1869) & $\mathrm{AM}, \mathrm{AP}$ \\
\hline & Phyllocycla pegasus (Selys, 1869) & AM, MT \\
\hline & Phyllocycla sordida (Selys, 1854) & $\mathrm{RO}$ \\
\hline & Phyllocycla volsella (Calvert, 1905) & AM \\
\hline & Phyllogomphoides andromeda (Selys, 1869) & AM, PA \\
\hline & Phyllogomphoides angularis Belle, 1982 & $A M, A P, R O$ \\
\hline & Phyllogomphoides annectens (Selys, 1869) & MT \\
\hline & Phyllogomphoides atlanticus (Belle, 1970) & AM \\
\hline & Phyllogomphoides audax (Hagen in Selys, 1854) & PA \\
\hline & Phyllogomphoides calverti (Kirby, 1897) & $\mathrm{PA}, \mathrm{RO}$ \\
\hline & Phyllogomphoides cepheus Belle, 1980 & $A M, A P, M T$ \\
\hline & Phyllogomphoides fuliginosus (Hagen in Selys, 1854) & PA \\
\hline & Phyllogomphoides major Belle, 1984 & AM \\
\hline & Phyllogomphoides pedunculus Belle, 1984 & AM \\
\hline & Phyllogomphoides praedatrix Belle, 1982 & RO \\
\hline & Phyllogomphoides pseudangularis Belle, 1994 & $\mathrm{AM}, \mathrm{AP}$ \\
\hline & Phyllogomphoides selysi (Navás, 1924) & AM \\
\hline & Phyllogomphoides spiniventris Belle, 1994 & MT \\
\hline & Phyllogomphoides suspectus Belle, 1994 & MT, RO \\
\hline & Phyllogomphoides undulatus (Needham, 1944) & $A M, A P, P A$ \\
\hline & Progomphus amazonicus Belle, 1973 & $A M, R R, R O$ \\
\hline & Progomphus angeloi Belle, 1994 & AM \\
\hline & Progomphus approximatus Belle, 1966 & AM \\
\hline & Progomphus boliviensis Belle, 1973 & AM \\
\hline & Progomphus delicatus Belle, 1973 & AM \\
\hline & Progomphus fassli Belle, 1973 & PA \\
\hline & Progomphus geijskesi Needham, 1944 & MT, PA \\
\hline & Progomphus guyanensis Belle, 1966 & AM \\
\hline & Progomphus intricatus Hagen in Selys, 1858 & $\mathrm{AM}, \mathrm{MT}, \mathrm{PA}$ \\
\hline & Progomphus maculatus Belle, 1984 & AM, PA \\
\hline & Progomphus nigellus Belle, 1990 & RO \\
\hline & Progomphus phylocramus Ris, 1918 & AM \\
\hline & Progomphus perithemoides Belle, 1980 & MT \\
\hline & Progomphus perpusillus Ris, 1918 & AM \\
\hline & Progomphus pijpersi Belle, 1966 & $\mathrm{AM}, \mathrm{RO}$ \\
\hline & Progomphus pygmaeus Selys, 1873 & MT, PA \\
\hline & Progomphus recticarinatus Calvert, 1909 & MT \\
\hline & Progomphus tibialis Belle, 1973 & AM \\
\hline & Zonophora batesi Selys, 1869 & $A M, A P, P A$ \\
\hline & Zonophora calippus Selys, 1869 & $A C, A M, P A, R O, T O$ \\
\hline & Zonophora nobilis Belle, 1983 & AM \\
\hline & Zonophora supratriangularis Schmidt, 1941 & AM \\
\hline & Zonophora surinamensis Needham, 1944 & AP \\
\hline & Zonophora wucherpfennigi Schmidt, 1941 & AM, PA \\
\hline \multicolumn{3}{|l|}{ Libellulidae } \\
\hline & Anatya guttata (Erichson in Schomburgk, 1848) & $A M, A P, M T, P A, R R$ \\
\hline & Antidythemis trameiformis Kirby, 1889 & $\mathrm{MT}, \mathrm{PA}$ \\
\hline
\end{tabular}


Table 1. Continue...

\begin{tabular}{|c|c|c|}
\hline Suborder/Family & Species & Brazilian Legal Amazon states \\
\hline \multirow[t]{50}{*}{ Libellulidae } & Argyrothemis argentea Ris, 1911 & $\mathrm{AM}, \mathrm{MT}, \mathrm{PA}, \mathrm{RO}$ \\
\hline & Brachymesia furcata (Hagen, 1861) & AC \\
\hline & Brachymesia herbida (Gundlach, 1889) & $A C, A M, A P, M T, P A, T O$ \\
\hline & Brechmorhoga mendax (Hagen, 1861) & MT \\
\hline & Brechmorhoga nubecula (Rambur, 1842) & $\mathrm{AM}, \mathrm{MT}$ \\
\hline & Brechmorhoga praecox Hagen, 1861 & $\mathrm{RR}$ \\
\hline & Brechmorhoga praedatrix Calvert, 1909 & $\mathrm{AM}, \mathrm{MT}$ \\
\hline & Brechmorhoga travassosi Santos, 1946 & MT \\
\hline & Dasythemis esmeralda Ris, 1910 & $\mathrm{AM}, \mathrm{MT}, \mathrm{PA}, \mathrm{RO}$ \\
\hline & Dasythemis essequiba Ris, 1919 & $\mathrm{AM}, \mathrm{PA}$ \\
\hline & Dasythemis venosa (Burmeister, 1839) & MT \\
\hline & Diastatops dimidiata (Linnaeus, 1758) & AP, PA \\
\hline & Diastatops emilia Montgomery 1940 & PA \\
\hline & Diastatops estherae Montgomery, 1940 & AM \\
\hline & Diastatops intensa Montgomery, 1940 & $\mathrm{AM}, \mathrm{MT}, \mathrm{PA}, \mathrm{RO}$ \\
\hline & Diastatops maxima Montgomery, 1940 & AM \\
\hline & Diastatops nigra Montgomery, 1940 & $A M, A P, R O$ \\
\hline & Diastatops obscura (Fabricius, 1775) & $A C, A M, A P, M A, M T, P A, R O, R R, T O$ \\
\hline & Diastatops pullata (Burmeister, 1839) & $A P, A M, M T, P A, R R$ \\
\hline & Dythemis nigra Martin, 1897 & AM, TO, MT \\
\hline & Dythemis sterilis Hagen, 1861 & $A C, P A, M T, R R$ \\
\hline & Elasmothemis cannacrioides (Calvert, 1906) & MT, PA, RR \\
\hline & Elasmothemis constricta (Calvert, 1898) & TO \\
\hline & Elasmothemis williamsoni (Ris, 1919) & AM, MT, PA \\
\hline & Elga leptostyla Ris, 1909 & $\mathrm{AM}, \mathrm{MT}, \mathrm{RO}$ \\
\hline & Erythemis attala (Selys in Sagra, 1857) & $A M, A P, P A, R O$ \\
\hline & Erythemis carmelita Williamson, 1923 & AM \\
\hline & Erythemis credula (Hagen, 1861) & $A C, M T, P A, R R$ \\
\hline & Erythemis haematogastra (Burmeister, 1839) & $A C, A M, A P, M A, M T, P A$ \\
\hline & Erythemis mithroides (Brauer in Therese, 1900) & $A C, A M, A P, P A$ \\
\hline & Erythemis peruviana (Rambur, 1842) & $\mathrm{AM}, \mathrm{AP}, \mathrm{MA}, \mathrm{MT}, \mathrm{PA}, \mathrm{RR}$ \\
\hline & Erythemis plebeja (Burmeister, 1839) & $A M, P A, M A, M T, R O$ \\
\hline & Erythemis vesiculosa (Fabricius, 1775) & $A C, A M, A P, M A, M T, P A, R R, R O, T O$ \\
\hline & Erythrodiplax abjecta (Rambur, 1842) & MT \\
\hline & Erythrodiplax acantha Borror, 1942 & TO \\
\hline & Erythrodiplax amazonica Sjöstedt, 1918 & $\mathrm{AM}, \mathrm{MT}, \mathrm{PA}, \mathrm{RO}$ \\
\hline & $\begin{array}{l}\text { Erythrodiplax ana Guillermo-Ferreira, Vilela, Del-Claro e } \\
\text { Bispo, } 2016\end{array}$ & MT \\
\hline & Erythrodiplax anatoidea Borror, 1942 & $\mathrm{AM}, \mathrm{RO}$ \\
\hline & Erythrodiplax angustipennis Borror, 1942 & RO \\
\hline & Erythrodiplax attenuata (Kirby, 1889) & $A C, A M, P A, M T, R R, R O$ \\
\hline & Erythrodiplax avittata Borror, 1942 & PA \\
\hline & Erythrodiplax basalis (Kirby, 1897) & $A C, A M, A P, M A, M T, P A, R R, R O$ \\
\hline & Erythrodiplax branconensis Sjöstedt, 1929 & AC \\
\hline & Erythrodiplax castanea (Burmeister, 1839) & AM, MA, MT, PA, RO, TO \\
\hline & Erythrodiplax clitella Borror, 1942 & $A C$ \\
\hline & Erythrodiplax famula (Erichson, 1848) & $A M, A P, M T, P A, R R, R O$ \\
\hline & Erythrodiplax fusca (Rambur, 1842) & $A C, A M, A P, M A, M T, P A, R R, R O, T O$ \\
\hline & Erythrodiplax juliana Ris, 1911 & $\mathrm{AM}, \mathrm{MT}, \mathrm{PA}, \mathrm{RR}, \mathrm{RO}, \mathrm{TO}$ \\
\hline & Erythrodiplax latimaculata Ris, 1911 & $A C, M A, M T, P A$ \\
\hline & Erythrodiplax lativittata Borror, 1942 & $\mathrm{AM}, \mathrm{MT}, \mathrm{RR}, \mathrm{RO}, \mathrm{TO}$ \\
\hline
\end{tabular}

To be continue... 
Table 1. Continue...

\begin{tabular}{|c|c|c|}
\hline Suborder/Family & Species & Brazilian Legal Amazon states \\
\hline \multirow[t]{50}{*}{ Libellulidae } & Erythrodiplax laurentia Borror, 1942 & $A M, A P$ \\
\hline & Erythrodiplax longitudinalis (Ris, 1919) & $A M, M T, P A, R O$ \\
\hline & Erythrodiplax lygaea Ris, 1911 & MT \\
\hline & Erythrodiplax luteofrons Santos, 1956 & TO \\
\hline & Erythrodiplax maculosa (Hagen, 1861) & MT, PA \\
\hline & Erythrodiplax melanica Borror, 1942 & $M A, P A, R O, T O$ \\
\hline & Erythrodiplax melanorubra (Borror, 1942) & MA \\
\hline & Erythrodiplax minuscula (Rambur, 1842) & MT \\
\hline & $\begin{array}{l}\text { Erythrodiplax nataliae Palacio, Muzon, Juen, Ferreira \& } \\
\text { Batista, } 2020\end{array}$ & MT \\
\hline & Erythrodiplax ochracea (Burmeister, 1834) & MT, PA \\
\hline & Erythrodiplax paraguayensis (Förster, 1905) & $\mathrm{AC}, \mathrm{MT}, \mathrm{PA}, \mathrm{RR}, \mathrm{RO}$ \\
\hline & Erythrodiplax solimaea Ris, 1911 & AM, PA \\
\hline & Erythrodiplax tenuis Borror, 1942 & RO \\
\hline & Erythrodiplax umbrata (Linnaeus, 1758) & $A C, A M, A P, M A, M T, P A, R R, R O$ \\
\hline & Erythrodiplax unimaculata (De Geer, 1773) & $\mathrm{AM}, \mathrm{PA}, \mathrm{MT}, \mathrm{RO}$ \\
\hline & Erythrodiplax venusta (Kirby, 1897) & $A M, A P, M T, R R$ \\
\hline & Fylgia amazonica (Kirby, 1889) & $A C, A M, M A, M T, P A, R O$ \\
\hline & Gynothemis aurea Navás, 1933 & MT \\
\hline & Gynothemis pumila (Karsch, 1890) & $\mathrm{AM}, \mathrm{MT}, \mathrm{PA}, \mathrm{RO}$ \\
\hline & Gynothemis venipunctata Calvert in Ris, 1909 & MT, TO \\
\hline & Idiataphe amazonica (Kirby, 1889) & AM, MT, PA, RR \\
\hline & Idiataphe batesi (Ris, 1913) & $\mathrm{AM}, \mathrm{MT}, \mathrm{PA}, \mathrm{TO}$ \\
\hline & Idiataphe cubensis Scudder, 1866 & AM \\
\hline & Idiataphe longipes (Hagen, 1861) & $\mathrm{AM}, \mathrm{MT}, \mathrm{PA}, \mathrm{RO}$ \\
\hline & Libellula herculea Karsch, 1889 & AM \\
\hline & Macrothemis absimilis Costa, 1991 & PA \\
\hline & Macrothemis brevidens Belle, 1983 & AM \\
\hline & Macrothemis declivata Calvert, 1909 & MT \\
\hline & Macrothemis flavescens (Kirby, 1897) & $\mathrm{MT}, \mathrm{RR}$ \\
\hline & Macrothemis extensa Ris, 1913 & AM, PA \\
\hline & Macrothemis hemichlora (Burmeister, 1839) & MT \\
\hline & Macrothemis heteronycha (Calvert, 1909) & MA, MT, TO \\
\hline & Macrothemis idalia Ris, 1919 & AM \\
\hline & Macrothemis imitans Karsch, 1890 & MT, PA \\
\hline & Macrothemis inacuta (Calvert, 1898) & MA \\
\hline & Macrothemis ludia Belle, 1987 & PA \\
\hline & Macrothemis musiva Calvert, 1898 & MT, RO \\
\hline & Macrothemis newtoni Costa, 1990 & AM \\
\hline & Macrothemis nobilis Rácenis, 1957 & AM \\
\hline & Macrothemis pseudimitans Calvert, 1898 & MT \\
\hline & Macrothemis rupicola Rácenis, 1957 & AM \\
\hline & Macrothemis taurepan De Marmels, 2008 & AM \\
\hline & Macrothemis ultima González, 1992 & MT \\
\hline & Miathyria marcella (Selys in Sagra, 1857) & $A M, A P, M A, M T, P A, R R$ \\
\hline & Miathyria simplex (Rambur, 1842) & $A C, A M, A P, M T, P A, R R, R O$ \\
\hline & Micrathyria aequalis (Hagen, 1861) & PA \\
\hline & Micrathyria almeidai Santos, 1945 & TO \\
\hline & Micrathyria artemis Ris, 1911 & $A C, A M, A P, M A, M T, P A, R O$ \\
\hline & Micrathyria athenais Calvert, 1909 & MT \\
\hline & Micrathyria atra (Martin, 1897) & $A M, A P, M T, P A$ \\
\hline
\end{tabular}


Table 1. Continue...

\begin{tabular}{|c|c|c|}
\hline Suborder/Family & Species & Brazilian Legal Amazon states \\
\hline \multirow[t]{51}{*}{ Libellulidae } & Micrathyria cambridgei Kirby, 1897 & AM \\
\hline & Micrathyria catenata Calvert, 1909 & AM, MA, MT, PA \\
\hline & Micrathyria coropinae Geijskes, 1963 & MT \\
\hline & Micrathyria dido Ris, 1911 & PA, RO \\
\hline & Micrathyria divergens Westfall, 1992 & MA \\
\hline & Micrathyria dunklei Westfall, 1992 & RO \\
\hline & Micrathyria eximia Kirby, 1897 & $\mathrm{MA}, \mathrm{MT}, \mathrm{PA}, \mathrm{RO}$ \\
\hline & Micrathyria hesperis Ris, 1911 & PA, MT \\
\hline & Micrathyria hippolyte Ris, 1911 & $\mathrm{AM}, \mathrm{MT}$ \\
\hline & Micrathyria kleerekoperi Calvert, 1946 & RO \\
\hline & Micrathyria laevigata Calvert, 1909 & AM \\
\hline & Micrathyria longifasciata Calvert, 1909 & MT \\
\hline & Micrathyria mengeri Ris, 1919 & MA, MT, RO \\
\hline & Micrathyria occipita Westfall, 1992 & MT \\
\hline & Micrathyria ocellata Martin, 1897 & $A C, M A, M T, P A$ \\
\hline & Micrathyria paruensis Geijskes, 1963 & MA \\
\hline & Micrathyria pirassunungae Santos, 1953 & PA, MT \\
\hline & Micrathyria pseudeximia Westfall, 1992 & $\mathrm{AM}, \mathrm{MA}, \mathrm{MT}, \mathrm{PA}, \mathrm{RO}$ \\
\hline & Micrathyria pseudhypodidyma Costa, Lourenço \& Viera, 2002 & MT \\
\hline & Micrathyria romani Sjöstedt, 1918 & $A M, M A, P A$ \\
\hline & Micrathyria spinifera Calvert, 1909 & $A M, A P, M T, P A, R O$ \\
\hline & Micrathyria spuria (Selys in Therese, 1900) & MT, RR \\
\hline & Micrathyria stawiarskii Santos, 1953 & PA, MT \\
\hline & Micrathyria tibialis Kirby, 1987 & AM, MT, PA, RR \\
\hline & Micrathyria ungulata Förster, 1907 & PA, MT \\
\hline & Micrathyria venezuelae De Marmels, 1989 & AM \\
\hline & Misagria calverti Geijskes, 1951 & $\mathrm{AM}, \mathrm{RO}$ \\
\hline & Misagria divergens De Marmels, 1981 & AM \\
\hline & Misagria parana Kirby, 1889 & $A M, P A, R R, R O$ \\
\hline & Nephepeltia aequisetis Calvert, 1909 & MT \\
\hline & Nephepeltia berlai Santos, 1950 & MA, PA \\
\hline & Nephepeltia flavifrons (Karsch, 1889) & $A C, A P, P A, M A, M T, R O, R R$ \\
\hline & Nephepeltia flavipennis Von Ellenrieder, 2014 & RO \\
\hline & Nephepeltia leonardina Rácenis, 1953 & MT, RO \\
\hline & Nephepeltia phryne (Perty, 1834) & $A P, M A, M T, P A, R O, R R$ \\
\hline & Oligoclada abbreviata (Rambur, 1842) & $A M, M T, P A, R O, R R$ \\
\hline & Oligoclada amphinome Ris, 1909 & $\mathrm{AM}, \mathrm{PA}, \mathrm{MT}, \mathrm{RO}$ \\
\hline & Oligoclada borrori Santos, 1945 & AM \\
\hline & Oligoclada crocogaster Borror, 1931 & $\mathrm{PA}, \mathrm{RO}$ \\
\hline & Oligoclada monosticha Borror, 1981 & $\mathrm{AC}, \mathrm{AM}$ \\
\hline & Oligoclada nemesis (Ris, 1909) & AM \\
\hline & Oligoclada pachystigma Karsch, 1890 & $\mathrm{AM}, \mathrm{MT}, \mathrm{PA}, \mathrm{RO}$ \\
\hline & Oligoclada risi Geijskes, 1984 & AM \\
\hline & Oligoclada stenoptera Borror, 1931 & $M A, P A, R O$ \\
\hline & Oligoclada sylvia (Kirby, 1889) & $A M, P A, R O$ \\
\hline & Oligoclada waikinimae De Marmels, 1992 & PA \\
\hline & Oligoclada walkeri Geijskes, 1931 & $\mathrm{AM}, \mathrm{PA}, \mathrm{MT}, \mathrm{RO}$ \\
\hline & Oligoclada xanthopleura Borror, 1931 & $\mathrm{AM}, \mathrm{PA}, \mathrm{MT}, \mathrm{RO}$ \\
\hline & Orthemis aequilibris Calvert, 1909 & AM, MT, PA, RO \\
\hline & Orthemis ambinigra Calvert, 1909 & MA \\
\hline & Orthemis ambirufa Calvert, 1909 & PA, MT, TO \\
\hline
\end{tabular}


Table 1. Continue...

\begin{tabular}{|c|c|c|}
\hline Suborder/Family & Species & Brazilian Legal Amazon states \\
\hline \multirow[t]{48}{*}{ Libellulidae } & Orthemis anthracina De Marmels, 1989 & MT \\
\hline & Orthemis attenuata (Erichson in Schomburgk, 1848) & AM, MT, PA \\
\hline & Orthemis biolleyi Calvert, 1906 & $A C, A M, M A, M T, P A, R O$ \\
\hline & Orthemis celata Von Ellenrieder, 2012 & PA \\
\hline & Orthemis concolor Ris, 1919 & MT, PA \\
\hline & Orthemis cultriformis Calvert, 1899 & $A C, A M, A P, M A, M T, P A, R O$ \\
\hline & Orthemis discolor (Burmeister, 1839) & $\mathrm{AM}, \mathrm{MA}, \mathrm{MT}, \mathrm{PA}, \mathrm{RR}, \mathrm{RO}$ \\
\hline & Orthemis faaseni Von Ellenrieder, 2012 & $\mathrm{RO}$ \\
\hline & Orthemis ferruginea (Fabricius, 1775) & $A M, P A, R O$ \\
\hline & Orthemis flavopicta Kirby, 1889 & MT, RO \\
\hline & Orthemis schmidti Buchholz, 1950 & AM, MA \\
\hline & Orthemis sulphurata Hagen, 1868 & AP \\
\hline & Pantala flavescens (Fabricius, 1798) & $A C, A M, A P, P A, M T, R R, R O, T O$ \\
\hline & Perithemis bella Kirby, 1889 & $\mathrm{AM}, \mathrm{PA}$ \\
\hline & Perithemis cornelia Ris, 1910 & $A C, A M, P A, R O$ \\
\hline & Perithemis domitia (Drury, 1773) & MT \\
\hline & Perithemis electra Ris, 1930 & $A C, A M, M T, R O$ \\
\hline & Perithemis icteroptera (Selys in Sagra, 1857) & $\mathrm{RO}$ \\
\hline & Perithemis lais (Perty, 1834) & $A M, A P, M A, M T, P A, R R, R O$ \\
\hline & Perithemis tenera (Say, 1840) & $\mathrm{AM}, \mathrm{MT}, \mathrm{PA}, \mathrm{RO}$ \\
\hline & Perithemis parzefalli Hoffmann, 1991 & MT \\
\hline & Perithemis thais Kirby, 1889 & $A C, A M, A P, M A, P A, M T, R O$ \\
\hline & Planiplax arachne Ris, 1912 & PA \\
\hline & Planiplax erythropyga (Karsch, 1891) & MA \\
\hline & Planiplax machadoi Santos, 1949 & PA \\
\hline & Planiplax phoenicura Ris, 1912 & AM, MA, MT, PA \\
\hline & Rhodopygia cardinalis (Erichson in Schomburgk, 1848) & $A C, A M, A P, M T, P A, R O, R R$ \\
\hline & Rhodopygia geijskesi Belle, 1964 & $\mathrm{AM}, \mathrm{MT}, \mathrm{RO}, \mathrm{RR}$ \\
\hline & Rhodopygia hollandi Calvert, 1907 & AM, MT, RO \\
\hline & Sympetrum roraimae De Marmels, 1988 & $\mathrm{RR}$ \\
\hline & Tauriphila argo (Hagen, 1869) & $A C, P A$ \\
\hline & Tauriphila australis (Hagen, 1867) & AM, PA \\
\hline & Tholymis citrina Hagen, 1867 & $A M, A P, M T, P A$ \\
\hline & Tramea binotata (Rambur, 1842) & $A C, A M, A P, M A, M T, P A$ \\
\hline & Tramea darwini Kirby, 1889 & $A M, A P, P A, R O$ \\
\hline & Tramea cophysa Hagen, 1867 & AC, MT, PA \\
\hline & Tramea minuta De Marmels \& Rácenis, 1982 & $\mathrm{AM}, \mathrm{AP}, \mathrm{MA}, \mathrm{MT}, \mathrm{PA}, \mathrm{RO}$ \\
\hline & Tramea rustica De Marmels \& Rácenis, 1982 & $A C, A M, A P, R R, R O$ \\
\hline & Uracis fastigiata (Burmeister, 1839) & $\mathrm{AC}, \mathrm{AM}, \mathrm{AP}, \mathrm{PA}, \mathrm{MA}, \mathrm{MT}, \mathrm{RR}, \mathrm{RO}, \mathrm{TO}$ \\
\hline & Uracis imbuta (Burmeister, 1839) & $\mathrm{AC}, \mathrm{AM}, \mathrm{AP}, \mathrm{PA}, \mathrm{MA}, \mathrm{MT}, \mathrm{RR}, \mathrm{RO}, \mathrm{TO}$ \\
\hline & Uracis infumata (Rambur, 1842) & $A C, A M, P A, M T, R O$ \\
\hline & Uracis ovipositrix Calvert, 1909 & $\mathrm{AM}, \mathrm{AP}, \mathrm{PA}, \mathrm{MT}, \mathrm{RR}, \mathrm{RO}$ \\
\hline & Uracis reducta Fraser, 1946 & MT, RO \\
\hline & Uracis siemensi Kirby, 1897 & AC, AM, PA, MA, MT, RO, TO \\
\hline & Zenithoptera anceps Pujol-Luz, 1993 & $A M, M A, P A, R O$ \\
\hline & Zenithoptera fasciata (Linnaeus, 1758) & $A C, A M, A P, P A, M T, R O, R R$ \\
\hline & Zenithoptera lanei Santos, 1941 & $\mathrm{AC}, \mathrm{AM}, \mathrm{MT}, \mathrm{MA}, \mathrm{PA}, \mathrm{RO}, \mathrm{TO}$ \\
\hline & Zenithoptera viola Ris, 1910 & $\mathrm{AM}, \mathrm{AP}, \mathrm{MT}, \mathrm{PA}$ \\
\hline \multicolumn{3}{|l|}{ Synthemistidae } \\
\hline & Neocordulia androgynis (Selys, 1871) & MT \\
\hline & Neocordulia batesi Selys, 1871 & AM \\
\hline
\end{tabular}


Table 1. Continue...

\begin{tabular}{lll}
\hline \multicolumn{1}{c}{ Suborder/Family } & \multicolumn{1}{c}{ Species } & Brazilian Legal Amazon states \\
\hline Synthemistidae & Neocordulia setifera (Hagen in Selys, 1871) & MT \\
& Neocordulia volxemi (Selys, 1874) & MT \\
\hline
\end{tabular}

Table 2. Species diversity on Brazilian Legal Amazon states. Acre- Ac, Amazonas -AM, Amapá- AP, Maranhão- MA, Mato Grosso- MT, Pará- PA, Rondônia-RO, Roraima- RR e Tocantins- TO.

\begin{tabular}{|c|c|c|c|c|c|c|c|c|c|}
\hline \multirow{2}{*}{$\begin{array}{l}\text { Suborder/Famliy } \\
\text { Zygoptera }\end{array}$} & \multicolumn{9}{|c|}{ Number of species per state } \\
\hline & AC & AM & AP & MA & MT & PA & RO & $\mathbf{R} \mathbf{R}$ & TO \\
\hline Calopterygidae Selys in Selys \& Hagen, 1850 & 04 & 15 & 05 & 06 & 17 & 15 & 14 & 03 & 02 \\
\hline Coenagrionidae Kirby, 1890 & 26 & 114 & 47 & 22 & 84 & 116 & 69 & 24 & 19 \\
\hline Dicteriadidae Montgomery, 1959 & 01 & 02 & 01 & 00 & 01 & 02 & 01 & 00 & 00 \\
\hline Heteragrionidae Rácenis, 1959 & 01 & 11 & 04 & 00 & 02 & 06 & 07 & 01 & 00 \\
\hline Lestidae Calvert, 1901 & 00 & 03 & 00 & 02 & 07 & 00 & 02 & 03 & 00 \\
\hline Megapodagrionidae Tillyard, 1917 & 00 & 01 & 00 & 00 & 01 & 01 & 01 & 00 & 00 \\
\hline Perilestidae Kennedy, 1920 & 01 & 08 & 02 & 01 & 01 & 06 & 06 & 01 & 00 \\
\hline Philogeniidae Rácenis, 1959 & 00 & 02 & 00 & 00 & 00 & 00 & 00 & 00 & 00 \\
\hline Platystictidae Kennedy, 1920 & 00 & 01 & 01 & 00 & 00 & 00 & 00 & 00 & 00 \\
\hline Polythoridae Munz, 1919 & 04 & 11 & 01 & 00 & 01 & 04 & 03 & 00 & 00 \\
\hline Rimanellidae Davies \& Tobin, 1984 & 00 & 01 & 00 & 00 & 00 & 00 & 00 & 00 & 00 \\
\hline \multicolumn{10}{|l|}{ Anisoptera } \\
\hline Aeshnidae Leach in Brewster, 1815 & 05 & 32 & 08 & 01 & 18 & 20 & 08 & 05 & 00 \\
\hline Corduliidae Selys in Selys \& Hagen, 1850 & 00 & 04 & 00 & 00 & 04 & 02 & 01 & 00 & 01 \\
\hline Gomphidae Rambur, 1842 & 01 & 47 & 10 & 02 & 22 & 27 & 13 & 03 & 04 \\
\hline Libellulidae Leach in Brewster, 1815 & 39 & 111 & 40 & 48 & 124 & 111 & 81 & 39 & 23 \\
\hline Synthemistidae Tillyard, 1911 & 00 & 01 & 00 & 00 & 03 & 00 & 00 & 00 & 00 \\
\hline Total number of species & 82 & 364 & 119 & 82 & 285 & 310 & 206 & 79 & 49 \\
\hline
\end{tabular}

data collected during the present study, 40 new records were added to the fauna of the state. With the current 364 confirmed species, the Amazonas state is the richest state in Odonata species in Brazil. The second greatest diversity in the country is found outside the Amazon region, more precisely in the state of Minas Gerais, which has 323 species recorded (VILELA 2021). This large richness found in Minas Gerais state is due probably to the environmental heterogeneity of the state, since, according to PAULSON (2006), Odonata species are distributed in a wide variety of habitats. Apart from its environmental conditions, the odonatofauna of Minas Gerais has been intensively studied in recent years (AMORIM et al. 2018; Barbosa et al. 2019; Ávila JúNIOR et al. 2020; VILELA et al. 2020, VENÂNCIO et al. 2021; VILELA 2021) which has also considerably increased knowledge of the order in the state. It is believed that the state of Pará, due to its dimensions and natural characteristics, has the possibility of occupying the second largest diversity of dragonflies in Brazil. Perhaps this has not happened yet due to the peculiarities existing in the Amazon, such as difficulty in transport, lack of infrastructure in many places, in addition to the complexity of forms of communication (Molento \& VIEIRA 2016). Thus, those factors are directly linked to the low species numbers found for some of the studied states.

With the exception of the states of Amazonas and Pará, which have consolidated large groups of studies on aquatic insects, the other states in the Legal Amazon still publish few studies on this topic. Probably the low diversities found for the states of Acre $(n=82)$, Maranhão $(n=82)$, Roraima $(n=79)$ and Tocantins $(n=49)$ (Table 2 ) are due to this lack of laboratories centered on the odontofauna studies. According to DE MARCO \& VIANNA (2005), the areas of the states of Tocantins and Maranhão were practically untouched by researchers. Even with the recent increase in studies addressing odonatofauna
(BAstos et al. 2019; Moura et al. 2020), these states are still undersampled. For example, Tocantins, with 49 species, comprised approximately $8 \%$ of the organisms recorded during the study; however, it is possible that the species richness for the state is much higher, because samplings were only made in very few locations.

Another poorly explored area, according to DE MARCO \& VIANNA (2005), is the Guiana shield region, especially in the states of Amapá and Roraima. In recent studies published by GARCIA JUNIOR et al. (2020, 2021a, 2021b) 58 new records of Odonata were added to the state of Amapá, increasing the number of confirmed occurrences by $48 \%$. Roraima, with 79 species, had the second lowest diversity among the states in the Legal Amazon, results that indicate that the shield region is still underexplored.

According to VIEIRA et al. (2005) deforestation is the main consequence of the biodiversity loss in the Amazon. In addition, many species in this environment have small populations, which makes these organisms very sensitive to changes in habitats (THIOLLAY 1994). According to CLAUSNITZER et al. (2009), about $10 \%$ of the world's species of Odonata are in some category of extinction risk, which reinforces the urgency of recognizing the species, mainly from the Amazon region, before the areas are totally affected by anthropic action. In conclusion, the elaboration of inventories and the construction of catalogs will play a fundamental role in the knowledge of biodiversity of the Amazon region, also helping to carry out new initiatives aimed at recognizing fauna and preserving certain areas.

\section{ACKNOWLEDGEMENTS}

This study was funded by the Coordenação de Aperfeiçoamento de Pessoal de Nível Superior (CAPES) and 
the Fundação de Amparo à Pesquisa do Amapá (FAPEAP). DSV thanks the Fundação de Amparo à Pesquisa do Estado de São Paulo (FAPESP) for the postdoctoral fellowship (Proc. 2019/26438-9).

\section{REFERENCES}

Alves-Martins, F, LS Brasil, L Juen, PJR De Marco, J Stropp \& J Hortal, 2019. Metacommunity patterns of Amazonian Odonata: the role of environmental gradients and major rivers. PeerJ, 7: e6472. DOI: https://doi.org/10.7717/ peerj. 6472

Amorim, MS, MM Souza \& CS Anjos, 2018. Riqueza de libélulas (Insecta: Odonata) no município de Bueno Brandão, sul de Minas Gerais. MG. Biota, 11: 16-32.

Ávila Júnior, WF, GLV Machado, FAA Lencioni \& MAA Carneiro, 2020. Distribution and composition of Dragonfly and Damselfly species (Odonata) of the upper Rio das Velhas, Ouro Preto, Minas Gerais State, Brazil. Papéis Avulsos de Zoologia, 60: e20206065. DOI: https://doi.org/10.11606/1807-0205/2020.60.65

Barbosa, MS, LR Borges, DS Vilela, H Venâncio \& JC Santos, 2019. Odonate Communities of the Sucupira Reservoir, Rio Uberabinha, Minas Gerais, Brazil. Papéis Avulsos de Zoologia, 59: e20195922.DOI: https://doi.org/10.11606/1807-0205/2019.59.22

Bastos, RC, LS Brasil, FG Carvalho, LB Calvão, JOA Silva \& L Juen, 2019. Odonata of the state of Maranhão, Brazil: Wallacean shortfall and priority areas for faunistic inventories. Biota Neotropica, 19: e20190734. https://doi.org/10.1590/1676-0611-BN-2019-0734

Brasil, LS, AFA De Andrade, BR Ribeiro, ZA Spigoloni, L Juen \& PJR De Marco, 2021. A nichebased gap analysis for the conservation of odonate species in the Brazilian Amazon. Aquatic Conserv: Marine and Freshwater Ecosystems, 31: 1150-1157. DOI: https://doi.org/10.1002/aqc.3599

Brito, JP, FG Carvalho \& L Juen, 2021. Response of the Zygopteran Community (Odonata: Insecta) to Change in Environmental Integrity Driven by Urbanization in Eastern Amazonian Streams. Ecologies, 2: 150-163. DOI: https://doi.org/10.3390/ecologies2010008

Calvão, LB, PJR De Marco \& JD Batista, 2014. Odonata (Insecta) from Nova Xavantina, Mato Grosso, Central Brazil: Information on species distribution and new records. Check List, 10: 299-307. DOI: https://doi.org/10.15560/10.2.299

Clausnitzer, V, VJ Kalkman, M Ramc, B Collen, JEM Baillie, M Bedjanic, WRT Darwall, KDB Dijkstra, R Dowf, J Hawking, H Karube, E Malikova, D Paulson, K Schutte, F Suhling, RJ Villanuevam, NV Ellenrieder \& K Wilson, 2009. Odonata enter the biodiversity crisis debate: the first global assessment of an insect group. Biological Conservation, 142: 1864-1869. DOI: https://doi.org/10.1016/j. biocon.2009.03.028

Corbet, PS, 1999. Dragonflies: behavior and ecology of Odonata. Ithaca: Comstock.

De Marco, PJR \& DM Vianna, 2005. Distribuição do esforço de coleta de Odonata no Brasil subsídios para escolha de áreas prioritárias para levantamentos faunísticos. Lundiana, 6:13-26.

De Marco, PJR, JD Batista \& HSR Cabette, 2015. Community Assembly of Adult Odonates in Tropical Streams: An Ecophysiological Hypothesis. PLoS ONE, 10: e0123023. DOI: https://doi.org/10.1371/journal.pone.0123023

Garcia Junior, MDN, MTS Damasceno, MJL Martins, TS Costa, RMA Ferreira \& RNP Souto, 2020. New records of dragonflies and damselflies (Insecta: Odonata) from Amapá state, Brazil. Biota Neotropica, 21: e20201074. DOI: https://doi.org/10.1590/1676-0611-BN-2020-1074

Garcia Junior, MDN, MTS Damasceno \& RNP Souto, 2021b. Novos Registros da família Aeshnidae
(Odonata: Anisoptera) para o estado do Amapá, Brasil. Nature and Conservation, 14(1): 181-184. DOI: https://doi.org/10.6008/CBPC2318-2881.2021.001.0020

Garcia Junior, MDN, MTS Damasceno, TS Costa \& RNP Souto, 2021a. Data from diversity of Zygoptera (Odonata) in the state of Amapá, Brazil. Anales de Biología, 43: 101-109. DOI: https://doi.org/10.6018/analesbio.43.10

GBIF, 2021. Global Biodiversity Information Facility. Available in: <https://www.gbif.org/pt/>.

Koroiva, R, UG Neiss, G Fleck \& N Hamada, 2020. Checklist of dragonflies and damselflies (Insecta: Odonata) of the Amazonas state, Brazil. Biota Neotropica, 20: e20190877. DOI: https://doi.org/10.1590/1676-0611-BN-2019-0877

IBGE, 2021. Amazônia Legal, área de atuação da SUDAM.

INPE, 2020. Prodes Amazônia. Monitoramento do Desmatamento da Floresta Amazônica Brasileira por Satélite. Available in: <http://www.obt.inpe.br/OBT/ assuntos/programas/amazonia/prodes>.

Juen, L \& PJR De Marco, 2012. Dragonfly endemism in the Brazilian Amazon: Competing hypotheses for biogeographical patterns. Biodiversity and Conservation, 21: 3507-3521. DOI: https://doi.org/10.1007/s10531-0120377-0

Juen, L, JMB Oliveira-Júnior \& Y Shimano, 2014. Composição e riqueza de odonata (Insecta) em riachos com diferentes níveis de conservação em um ecótone CerradoFloresta Amazônica. Acta Amazonica, 44: 175-184. DOI: https://doi.org/10.1590/s0044-59672014000200008

Laurence, WF, MA Cochrane, S Bergen, PM Fearnside, P Delamônica, C Barber, S D'Angelo \& T Fernandes, 2001. The future of the Brazilian Amazon. Science, 291: 438-439.

Marcovitch, J \& V Pinsky, 2020. Bioma Amazônia: atos e fatos. Estudos Avançados, 34: 83-106. DOl: https://doi.org/10.1590/s0103-4014.2020.34100.007

Miguel, TB, JMB Oliveira-Junior, R Ligeiro, \& L Juen, 2017. Odonata (Insecta) as a tool for the biomonitoring of environmental quality. Ecological Indicators, 81: 555-566. DOI: https://doi.org/10.1016/j.ecolind.2017.06.010

Molento, FHB \& CRM Vieira, 2016. O risco na pesquisa de campo em saúde na Amazônia brasileira: mais de um século de desafios (1900-2015). Revista Pan- Amazônica de Saúde, 7: 231-244. DOI: https://doi.org/10.5123/S217662232016000500026

Moura, LP, SEM Couceiro, LJuen \& DS Veras, 2020. Congruence of the composition of Odonata between dry and rainy seasons in the Maranhense Cerrado. International Journal of Odonatology, 23: 305-314. DOI: https://doi.org/10.1080 /13887890.2020.1779826

Neiss, UG \& N Hamada, 2014. Ordem Odonata, pp. 217-282. In Hamada, N, JL Nessimian \& RB Querino (eds). Insetos aquáticos na Amazônia brasileira: taxonomia, biologia e ecologia. Editora do INPA, Manaus.

Oliveira-Júnior, JMB \& L Juen, 2019. The Zygoptera/Anisoptera ratio (Insecta: Odonata): A new tool for habitat alterations assessment in Amazonian streams, Neotropical Entomology, 48: 552-560. DOI: https://doi.org/10.1007/ s13744-019-00672-x

Paulson, DR, 2006. The importance of forests to neotropical dragonflies. In Cordero, R (Ed). Forests and Dragonflies, 4er WDA International Symposium of Odonatology, Pontevedra (Spain). pp.79-101.

Paulson, DR, M Scorr \& C Deliry, 2021. World Odonata List. Available in: <https://www2.pugetsound.edu/academics/ academic-resources/slater-museum/biodiversityresources/dragonflies/world-odonata-list2/>

Pinto, AP, 2020. Odonata. In: Taxonomic Catalog of Fauna of Brasil. PNUD. Available in: <http://fauna.jbrj.gov.br/fauna/ faunadobrasil/171>

Silva, JMC, AB Rylands \& GAB Fonseca, 2005. O destino das áreas de endemismo da Amazônia. Megadiversidade, 1: 124-131. 
$\mathrm{SiBBr}$ - Sistemas de Informações sobre a Biodiversidade, 2021. Available in: <https://www.sibbr.gov.br/>.

Thiollay, JM, 1994. Structure, density and rarity in an Amazonian rainforest bird community. Journal of Tropical Ecology, 10: 449-481. DOI: https://doi.org/10.1017/ s0266467400008154

Venâncio, H, DS Vilela, MS Barbosa \& JC Santos. 2021. Dragonflies and Damselflies in a region of the Triângulo Mineiro, Minas Gerais: checklist and taxonomic additions. Biota Neotropica, 21: e20201182. DOI: https://doi.org/10.1590/1676-0611-BN-2020-1182

Vieira, ICG, JMC Silva \& PM Toledo, 2005. Estratégias para evitar a perda de biodiversidade na
Amazônia. Estudos Avançados, 19: 153-164. DOI: https://doi.org/10.1590/s0103-40142005000200009

Vilela, DS, R Koroiva, THA Tosta, MC Novaes \& R GuillermoFerreira, 2020. Dragonflies and damselflies from the West of Minas Gerais, Brazil: checklist and new records. Biota Neotropica, 20: e20190851. DOI: https://doi.org/10.1590/1676-0611-bn-2019-0851

Vilela, DS, 2021. Libélulas de Minas Gerais. Available in: $<$ http://libelulasdemg.com.br>.

Von Ellenrieder, N, 2009. Databasing dragonflies: state of knowledge in the Neotropical region. Agrion, 13: 58-72.

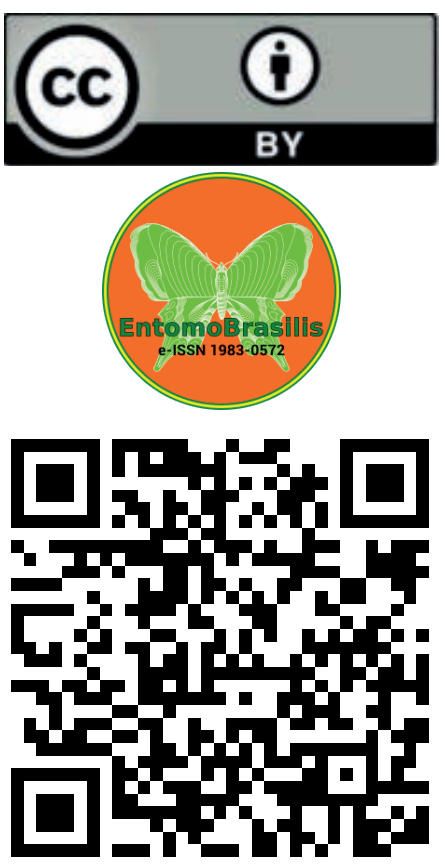

